\title{
Avaliação do Consumo de Energia do Protocolo de Roteamento RPL com Cenários de Perdas de nós na Rede.
}

\author{
Yelco Marante $^{1}$, Alex B. Vieira ${ }^{1}$, Edelberto Franco ${ }^{1}$, Frederico O. Sales ${ }^{1}$ \\ ${ }^{1}$ Departamento de Ciência da Computação - UFJF \\ \{yelcomarante, edelberto\}@ice.ufjf.br, alex.borges@ufjf.edu.br \\ frederico.sales@engenharia.ufjf.br
}

\begin{abstract}
The sensor nodes are low-cost electronic device able to selforganizing in low-power networks and susceptible to loss data packets, having computational and energy limitations. For these networks, the IETF standardized the Routing Protocol for Low Power and Lossy Networks (RPL), in which each secondary nodes select a better parent according to some objective functions (OFs). In this work, we propose the evaluation of the energy consumption of the RPL routing protocol in scenarios on lossy networks. We did simulations in the Contiki Cooja 3.0 considering the ETX and HOP metrics to evaluate three distinct topologies: tree, circular and mesh. Our results of the simulations show that the circular topology has the best (lowest) energy consumption, with 15\% better than the mesh topology and 30\% against the tree topology.
\end{abstract}

Resumo. Os nós sensores são dispositivos eletrônicos de custo módico capazes de se auto organizarem em redes de baixo consumo energético e suscetiveis a perdas de pacotes, possuem limitações computacionais e energéticas. Para essas redes, o IETF padronizou o protocolo de roteamento RPL (Protocolo de Roteamento para Redes de Baixa Potência e Perdas), no qual os nós secundários selecionam um melhor pai em relação ao nó receptor de acordo com algumas funções objetivos (OFs). Nesse trabalho propomos a avaliação do consumo energético do RPL com cenários de perdas de nós na rede. Nós fizemos simulações no Contiki Cooja 3.0 considerando as métricas ETX e HOP para avaliar três topologias: árvore, circular e em malha. Nossos resultados das simulações mostraram que a topologia circular teve o melhor (menor) consumo energético de $15 \%$ sob a topologia em malha e $30 \%$ sob a topologia árvore.

\section{Introdução}

As redes RSSFs apresentam questões desafiadoras relacionadas à confiabilidade da comunicação e ao uso eficiente da bateria do nó. Em particular, o roteamento é um problema crucial nas RSSFs, devido às possíveis falhas de comunicação, e limitada largura de banda e energia disponíveis. Desta forma, são necessárias técnicas sofisticadas para configurar e manter caminhos confiáveis, bem como para detectar prontamente falhas de link, sem desperdiçar energia e recursos de comunicação.

Nesse trabalho observamos a necessidade de se conhecer previamente determinadas características da topologia para a justificativa econômica e ecológica deste recurso. Assim, avaliamos o consumo energético do RPL com cenários de perdas de nós na rede. 
Nós fizemos simulações no Contiki Cooja 3.0 considerando as métricas ETX e HOP para avaliar três topologias: árvore, circular e em malha.

Os resultados mostram que o RPL tem excelente desempenho quanto a durabilidade da rede de sensores. Mais ainda, outro resultado significativo encontrado é com relação à influência da topologia no consumo energético da rede. A topologia circular apesar de ter restrições geográficas no sensoriamento se mostrou a melhor quanto ao consumo energético. Mais precisamente, a topologia circular teve o melhor (menor) consumo energético de $15 \%$ sob a topologia em malha e $30 \%$ sob a topologia árvore.

\section{Fundamentação Teórica}

\section{Redes de Sensores Sem Fio (RSSFs)}

Avanços recentes na comunicação sem fio e na eletrônica permitiram o desenvolvimento de nós de sensores multifuncionais de baixa potência e baixo custo. Esses sensores têm a capacidade de detectar, processar e enviar/receber informações em curtas distâncias. Em geral, estão presentes de forma densa, a fim de coletar dados de alta precisão e executar outras tarefas complexas relacionadas tanto à coleta quanto a disseminação de informações. Porém, deve-se levar em consideração suas limitações.

\section{Protocolo de Roteamento para Redes de Baixa Potência e Perdas (RPL)}

O RPL é um protocolo de roteamento pró-ativo leve e requer apenas pequenos recursos de energia e memória para operar, o que o torna uma excelente escolha para redes com limitações, como as da Internet das Coisas (IoT). O RPL é flexível e personalizável para adaptar os requisitos de aplicações IoT heterogêneas. Isso é possível com a ajuda de Funções de Objetivo (OF). As OFs se baseiam em métricas para indicar as melhores rotas existentes [Alvi et al. 2017]. As métricas de roteamento são selecionadas de acordo com a operação de rede ou demanda da aplicação. Elas tentam maximizar ou, em alguns casos, minimizar a OF para alcançar o melhor desempenho. Para evitar qualquer loop de roteamento, o RPL utiliza pesos associados à classificação de cada nó no grafo.

\section{Métricas de Roteamento}

A métrica de roteamento ETX (Expected Transmission Count) [Alvi et al. 2017] é baseada no número esperado de tentativas de transmissão de pacote de dados necessárias para transmitir com sucesso o pacote. É uma métrica baseada na qualidade do link que enfatiza a taxa de entrega de pacotes entre dispositivos de comunicação e não estatísticas de nível de nó. O ETX não considera o comprimento do caminho e pode selecionar caminhos mais longos. Além disso, as condições do canal são dinâmicas e é por isso que a comutação frequente dos pais é altamente provável. Já na métrica de roteamento HOP a decisão de seleção pai preferencial é baseada no número de saltos entre o nó emissor e o nó coletor. Essa métrica seleciona o caminho com um número mínimo de saltos. Claramente, essa é uma métrica baseada em rede que tenta enfatizar o número de saltos, em vez de estatísticas de nó ou link [Alvi et al. 2017].

\section{Proposta}

Os testes são implementados usando o simulador Cooja do Instant Contiki 3.0, utilizando as duas funções objetivos: ETX (MRHOF) [Gnawali and Levis 2012] e HOP (OF0) [Thubert 2012]. No Contiki, o uso do HOP resulta no uso de contagem de saltos e o uso de 
ETX que é baseada na qualidade dos links, as duas estão disponíveis no simulador Cooja. Neste estudo a abordagem inovadora será de utilizar faixas de transmissão/interferência nas topologias e em cada cenário tirar um percentil da quantidade de nós da rede para avaliar a resiliência e o consumo de energia. Esse percentil será simulando a falha de nós de maneira aleatória quem tiver um alto consumo de energia e não seja um nó sem filho. Nos testes há três topologias de rede distintas: árvore, circular e em malha. Cada cenário é executado durante 10 minutos e com duas faixas de transmissão/interferência diferentes. O projeto foi realizado utilizando um planejamento fatorial completo com as combinações possíveis em todos os níveis de todos os fatores. Um estudo de desempenho com $k$ fatores, com o fator $i$ tendo $n_{i}$ níveis, requer $n$ experimentos, onde: $n=\prod_{i=1}^{k} n_{i}$

\section{Avaliação}

Os parâmetros para os testes são mostrados na tabela 1. Nove testes são realizados com diferentes topologia, número de nós e faixa de transmissão/interferência.

Tabela 1. Parâmetros dos Testes.
\begin{tabular}{|l|c|l|}
\hline \multicolumn{2}{|c|}{ Parâmetros } & \multicolumn{2}{|c|}{ Valores } \\
\hline Funções de Objetivos & OF0 & MRHOF \\
\hline Métricas & HOP & ETX \\
\hline Faixa de Transmissão/Interferência & \multicolumn{2}{|c|}{$50 / 100 \mathrm{~m}, 70 / 90 \mathrm{~m}$} \\
\hline Topologias & Árvore, Circular, Malha \\
\hline Tempo da Simulação & \multicolumn{2}{|c|}{$10 \mathrm{~min}$} \\
\hline Número de Nós & \multicolumn{2}{|c|}{$10,20 \mathrm{e} 30$} \\
\hline Tipo de Nós & \multicolumn{2}{|c|}{ Sky Mote } \\
\hline Modo do canal sem fio & \multicolumn{2}{|c|}{ UDGM } \\
\hline
\end{tabular}

\subsection{Resultados dos testes}

Os cenários simulados nas topologias em árvore são mostrados na Figura 1; circular, na Figura 2; e em malha são mostrados na Figura 3. As médias dos consumos de energia: na topologia em árvore podem ser observados na Tabela 2, na topologia circular podem ser observados na Tabela 3 e na topologia em malha podem ser observados na Tabela 4.

\section{Topologia em Árvore}

A métrica ETX com 50/100m teve o maior consumo de energia nos cenários das perdas de nós, consumindo mais de $25 \%$ da energia a partir dos cenários superiores de $20 \%$ de perdas de nós. O que pode ser observado em relação às métricas é que ambas apresentam melhor desempenho na faixa de transmissão/interferência 70/90m, com o menor consumo de energia em todos os cenários das perdas de nós do HOP com 70/90m. À medida que o percentil de perdas de nós aumenta, as duas métricas aumentam ligeiramente o consumo de energia, mas a métrica ETX aumenta em maior proporção.

(a) Teste 1: 10 nós.

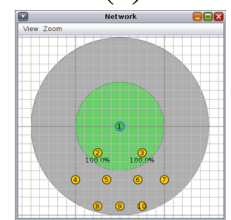

(b) Teste 2: 20 nós.

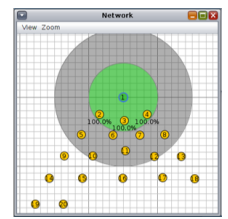

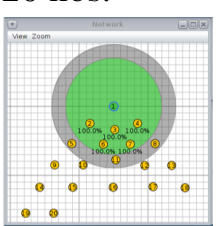

(c) Teste 3: 30 nós.

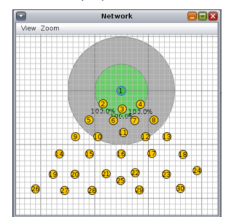

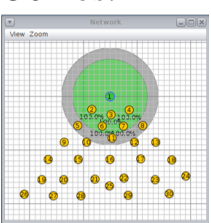

Figura 1. Topologia em árvore. (E) 50/100m - (D) 70/90m. 
Tabela 2. Consumo de Energia na topologia em árvore.

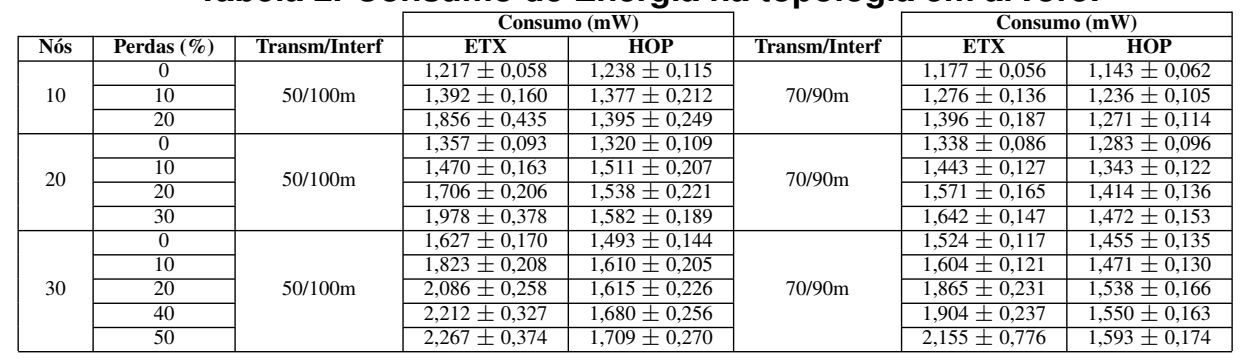

\section{Topologia Circular}

A métrica ETX com 50/100m teve o maior consumo de energia nos cenários das perdas de nós, com exceção no caso de 10 nós que o maior consumo foi da métrica ETX com 70/90m porque a topologia fez que todos os nós se comunicaram com o nó sink então com 70/90m teve maior consumo pelo uso da potencia de transmissão. O que pode ser observado em relação às métricas é que o menor consumo de energia foi em todos os cenários das perdas de nós do HOP com 70/90m. À medida que o percentil de perdas de nós aumenta, as duas métricas aumentam ligeiramente o consumo de energia, mas a métrica ETX aumenta em maior proporção nos casos de 20 e 30 nós.

(a) Teste 4: 10 nós.

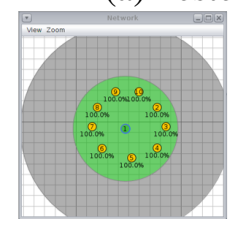

(b) Teste 5: 20 nós.

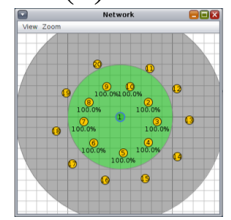

(c) Teste 6: 30 nós.

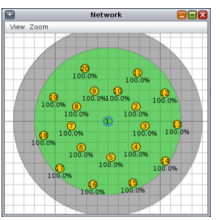

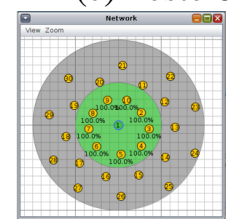

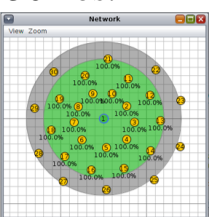

Figura 2. Topologia circular. (E) 50/100m - (D) 70/90m.

Tabela 3. Consumo de Energia na topologia circular.

\begin{tabular}{|c|c|c|c|c|c|c|c|}
\hline \multirow{2}{*}{ Nós } & \multicolumn{2}{|c|}{ 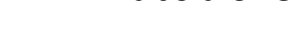 } & \multicolumn{2}{|c|}{ Consumo $(\mathrm{mW})$} & \multirow{2}{*}{ Transm/Interf } & \multicolumn{2}{|c|}{ Consumo (mW) } \\
\hline & Perdas (\%) & Transm/Interf & ETX & HOP & & ETX & HOP \\
\hline \multirow{3}{*}{10} & 0 & \multirow{3}{*}{$50 / 100 \mathrm{~m}$} & $1,035 \pm 0,031$ & $0,980 \pm 0,015$ & \multirow{3}{*}{ 70/90m } & $1,071 \pm 0,021$ & $1,032 \pm 0,014$ \\
\hline & 10 & & $1,054 \pm 0,045$ & $0,979 \pm 0,020$ & & $1,117 \pm 0,053$ & $1,028 \pm 0,014$ \\
\hline & 20 & & $1,089 \pm 0,033$ & $0,979 \pm 0,020$ & & $1,122 \pm 0,055$ & $1,023 \pm 0,013$ \\
\hline \multirow{4}{*}{20} & 0 & \multirow{4}{*}{$50 / 100 \mathrm{~m}$} & $1,214 \pm 0,043$ & $1,136 \pm 0,038$ & \multirow{4}{*}{ 70/90m } & $1,1030,028$ & $1,068 \pm 0,022$ \\
\hline & 10 & & $1,305 \pm 0,063$ & $1,206 \pm 0,071$ & & $1,157 \pm 0,044$ & $1,061 \pm 0,020$ \\
\hline & 20 & & $1,525 \pm 0,191$ & $1,247 \pm 0,100$ & & $1,165 \pm 0,004$ & $1,056 \pm 0,023$ \\
\hline & 30 & & $1,885 \pm 0,352$ & $1,263 \pm 0,107$ & & $1,188 \pm 0,050$ & $1,050 \pm 0,026$ \\
\hline \multirow{5}{*}{30} & 0 & \multirow{5}{*}{$50 / 100 \mathrm{~m}$} & $1,591 \pm 0,120$ & $1,278 \pm 0,061$ & \multirow{5}{*}{$70 / 90 \mathrm{~m}$} & $1,277 \pm 0,036$ & $1,177 \pm 0,040$ \\
\hline & 10 & & $1,723 \pm 0,154$ & $1,362 \pm 0,079$ & & $1,369 \pm 0,067$ & $1,234 \pm 0,049$ \\
\hline & 20 & & $1,917 \pm 0,240$ & $1,375 \pm 0,078$ & & $1,477 \pm 0,080$ & $1,250 \pm 0,053$ \\
\hline & 40 & & $1,977 \pm 0,266$ & $1,390 \pm 0,096$ & & $1,548 \pm 0,095$ & $1,279 \pm 0,057$ \\
\hline & 50 & & $2,075 \pm 0,299$ & $1,456 \pm 0,148$ & & $1,697 \pm 0,180$ & $1,302 \pm 0,066$ \\
\hline
\end{tabular}

\section{Topologia em Malha}

A métrica ETX com 50/100m teve o maior consumo de energia nos cenários das perdas de nós. No caso da simulação de 10 nós houve pouca diferença dos resultados em comparação com as simulações de 20 e 30 nós. O que pode ser observado em relação às métricas é que ambas apresentam melhor desempenho na faixa de transmissão/interferência 70/90m, com o menor consumo de energia em todos os cenários das perdas de nós do HOP com 70/90m. À medida que o percentil de perdas de nós aumenta, as duas métricas aumentam ligeiramente o consumo de energia, mas a métrica ETX aumenta em maior proporção, com exceção no caso de 10 nós. 
(a) Teste 4: 10 nós.

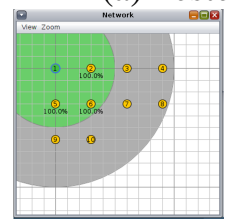

(b) Teste 5: 20 nós.

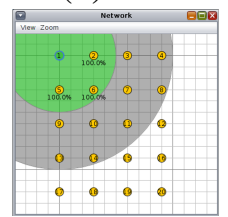

(c) Teste 6: 30 nós.

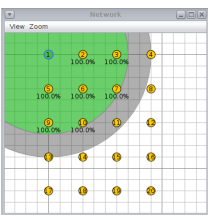

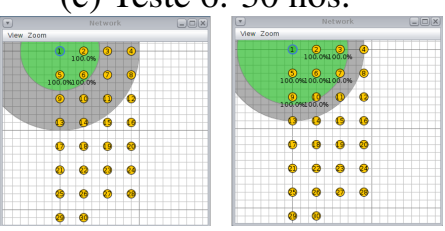

Figura 3. Topologia em malha. (E) 50/100m - (D) 70/90m.

Tabela 4. Consumo de Energia na topologia em malha.

\begin{tabular}{|c|c|c|c|c|c|c|c|}
\hline \multirow{2}{*}{ Nós } & \multirow[b]{2}{*}{ Perdas (\%) } & \multirow{2}{*}{ Transm/Interf } & \multicolumn{2}{|c|}{ Consumo $(\mathrm{mW})$} & \multirow{2}{*}{ Transm/Interf } & \multicolumn{2}{|c|}{ Consumo (mW) } \\
\hline & & & ETX & HOP & & ETX & HOP \\
\hline \multirow{3}{*}{10} & 0 & \multirow{3}{*}{$50 / 100 \mathrm{~m}$} & $1,212 \pm 0,100$ & $1,170 \pm 0,081$ & \multirow{3}{*}{ 70/90m } & $1,174 \pm 0,115$ & $1,091 \pm 0,066$ \\
\hline & 10 & & $1,284 \pm 0,122$ & $1,281 \pm 0,152$ & & $1,269 \pm 0,164$ & $1,180 \pm 0,141$ \\
\hline & 20 & & $1,448 \pm 0,256$ & $1,224 \pm 0,077$ & & $1,286 \pm 0,134$ & $1,189 \pm 0,155$ \\
\hline \multirow{4}{*}{20} & 0 & \multirow{4}{*}{$50 / 100 \mathrm{~m}$} & $1,548 \pm 0,135$ & $1,386 \pm 0,118$ & \multirow{4}{*}{$70 / 90 \mathrm{~m}$} & $1,297 \pm 0,061$ & $1,257 \pm 0,068$ \\
\hline & 10 & & $2,186 \pm 0,346$ & $1,557 \pm 0,163$ & & $1,472 \pm 0,154$ & $1,421 \pm 0,088$ \\
\hline & 20 & & $2,215 \pm 0,445$ & $1,492 \pm 0,134$ & & $1,561 \pm 0,149$ & $1,405 \pm 0,086$ \\
\hline & 30 & & $1,994 \pm 0,352$ & $1,425 \pm 0,094$ & & $1,670 \pm 0,239$ & $1,399 \pm 0,096$ \\
\hline \multirow{5}{*}{30} & 0 & \multirow{5}{*}{$50 / 100 \mathrm{~m}$} & $2,354 \pm 0,308$ & $1,590 \pm 0,159$ & \multirow{5}{*}{ 70/90m } & $1,458 \pm 0,076$ & $1,363 \pm 0,074$ \\
\hline & 10 & & $2,383 \pm 0,298$ & $1,817 \pm 0,234$ & & $1,589 \pm 0,172$ & $1,466 \pm 0,066$ \\
\hline & 20 & & $2,480 \pm 0,427$ & $1,754 \pm 0,197$ & & $1,633 \pm 0,117$ & $1,427 \pm 0,068$ \\
\hline & 40 & & $2,566 \pm 0,473$ & $1,701 \pm 0,212$ & & $1,699 \pm 0,111$ & $1,395 \pm 0,068$ \\
\hline & 50 & & $2,582 \pm 0,401$ & $1,653 \pm 0,157$ & & $1,751 \pm 0,149$ & $1,416 \pm 0,077$ \\
\hline
\end{tabular}

\subsection{Avaliação dos Testes}

Para avaliar as diferentes topologias utilizadas se aplicou a comparação mediante o método das observações pareadas utilizando um nível de confiança $=95 \%$ para poder concluir qual é a melhor métrica em cada cenário apresentado. Nos 6 cenários simulados da topologia em árvore, os resultados mostraram que a métrica HOP foi equivalente em 3 cenários (10 nós com 50/100m e 70/90m, 20 nós com 50/100m) e melhor em 3 cenários (20 nós com 70/90m, 30 nós com 50/100m e 70/90m). Nos 6 cenários simulados da topologia circular, os resultados mostraram que a métrica HOP foi equivalente em 2 cenários (10 nós com 70/90m, 20 nós com 50/100m) e melhor em 4 cenários (10 nós co 50/100m, 20 nós com 70/90m, 30 nós com 50/100m e 70/90m). Nos 6 cenários simulados da topologia em malha, os resultados mostraram que a métrica HOP foi equivalente em 2 cenários (10 nós com 50/100m, 20 nós com 70/90m) e melhor em 4 cenários (10 nós com 70/90m, 20 nós com 50/100m, 30 nós com 50/100m e 70/90m).

Após as comparações das topologias separadamente, foi feita a comparação da topologia árvore com a circular. Nesse caso as comparações foram feitas só com a métrica HOP que foi o resultado da melhor métrica nas avaliações feitas por cada topologia separada. Os resultados foram dos 6 cenários simulados a topologia circular foi melhor em todos os cenários. Seguidamente, foi feita a comparação da topologia circular com a malha. Igualmente as comparações foram feitas só com a métrica HOP. Os resultados foram dos 6 cenários diferentes a topologia circular foi melhor em todos os cenários.

\section{Trabalhos Relacionados}

Nós sensores têm energia de bateria limitada e podem estar em qualquer lugar, incluindo áreas de acesso difícil, não sendo possível recarregar ou substituir suas baterias. Portanto, o consumo de energia de cada nó do sensor deve ser minimizado para que a vida útil total da rede seja aumentada. Em [Husni et al. 2012] foi proposta uma técnica para melhorar o consumo de energia que funciona desligando os nós aleatoriamente de acordo com os critérios e condições definidas. Foi feito usando o simulador NS2 com o protocolo de roteamento AODV e uma certa quantidade fixa de nós, mas não considerou fazer 
as simulações variando a quantidade de nós nem mudando a topologia. Utilizando o simulador Cooja, existem vários trabalhos feitos em termos de desempenho de métricas, enquanto as métricas ETX e HOP estão disponíveis, somente a métrica ETX é usada na maioria dos estudos relacionados. [Thomson et al. 2016] fez a avaliação de desempenho das métricas ETX, HOP e Energia do RPL em ambientes com faixas de transmissão estendidas variando a quantidade de nós, a topologia e as faixas de transmissão/interferência. Mas, esse trabalho não considerou avaliar o consumo de energia através da resiliência da rede como foi apresentado por esta pesquisa.

\section{Conclusões e Trabalhos Futuros}

Apresentamos uma avaliação de desempenho do consumo de energia do protocolo de roteamento RPL, usando o simulador Cooja 3.0. Para as simulações foram realizados testes com cenários distintos em relação à topologia, quantidade de nós, faixa de transmissão/interferência e métrica especifica e as comparações foram feitas com um nível de confiança de $95 \%$. Destacamos como principal contribuição a técnica de se obter um certo percentual de nós uma vez que a rede já está estabelecida para observar seu comportamento. Com base nos resultados obtidos, observamos o melhor desempenho do consumo de energia da rede utilizando uma topologia circular com a métrica HOP. Nossos resultados estatísticos foram alcançados através do método das comparações pareadas e indicaram o seguinte: a topologia em árvore, circular e em malha com a métrica HOP teve o menor consumo de energia em comparação com a métrica ETX. Quando avaliadas em conjunto, a topologia circular teve o menor consumo de energia em comparação com as outras topologias com a métrica HOP. Com relação às três topologias utilizadas nos testes, pode-se concluir que uma topologia circular resulta em melhor desempenho em relação à manutenção do número de nós. Já uma topologia de árvore exige consideravelmente mais dos nós do primeiro salto, e também pode causar outros gargalos na rede. Trabalhos futuros incluem a criação de novas métricas para o RPL considerando os métodos de descoberta e ciência de topologia em redes sem fio.

\section{Agradecimentos}

Os autores agradecem o apoio de CAPES, CNPq, FAPEMIG, FAPERJ e FAPESP.

\section{Referências}

Alvi, S. A., ul Hassan, F., and Mian, A. N. (2017). On the energy efficiency and stability of rpl routing protocol. In IEEE IWCMC.

Gnawali, O. and Levis, P. (2012). The minimum rank with hysteresis objective function (No. RFC 6719).

Husni, H., Enzai, N. I. M., Rais, N. A. M., and Jusoh, Z. (2012). Evaluation of random node shutdown in wireless sensor network for improving energy efficiency. In IEEE BEIAC.

Thomson, C., Wadhaj, I., Romdhani, I., and Al-Dubai, A. (2016). Performance evaluation of RPL metrics in environments with strained transmission ranges. In IEEE AICCSA.

Thubert, P. (2012). Objective function zero for the routing protocol for low-power and lossy networks (RPL) (No. RFC 6552). 